\title{
Modular bootstrap, elliptic points, and quantum gravity
}

\author{
Ferdinando Gliozzi $\odot$ \\ Istituto Nazionale di Fisica Nucleare, Sezione di Torino, Dipartimento di Fisica, Università di Torino, \\ Via Pietro Giuria 1, 10125 Torino, Italy
}

(Received 2 September 2019; accepted 26 February 2020; published 17 March 2020)

\begin{abstract}
The modular bootstrap program for two-dimensional conformal field theories could be seen as a systematic exploration of the physical consequences of consistency conditions at the elliptic points and at the cusp of their torus partition function. The study at $\tau=i$, the elliptic point stabilized by the modular inversion $S$, was initiated by Hellerman [S. Hellerman, J. High Energy Phys. 08 (2011) 130], who found a general upper bound for the most relevant scaling dimension $\Delta$. Likewise, analyticity at $\tau=i \infty$, the cusp stabilized by the modular translation $T$, yields an upper bound on the twist gap. Here we study consistency conditions at $\tau=\exp (2 i \pi / 3)$, the elliptic point stabilized by $S T$. We find a much stronger upper bound in the large- $c$ limit, namely, $\Delta<\frac{c-1}{12}+0.092$, which is very close to the minimal mass threshold of the Bañados-Teitelboim-Zanelli black holes in the gravity dual of three-dimensional anti-de Sitter/two-dimensional conformal field theory correspondence.
\end{abstract}

DOI: 10.1103/PhysRevResearch.2.013327

\section{INTRODUCTION}

It is still an open question as to whether three-dimensional pure gravity exists as a quantum theory. In the case of a negative cosmological constant, according to holographic duality [1], solving pure quantum gravity means finding the two-dimensional (2D) conformal field theory (CFT) defined on the boundary of the asymptotically anti-de Sitter (AdS) space-time. At the classical level, pure 3D gravity is "trivial" in the sense that there are no gravitational waves; its degrees of freedom correspond to multitrace composites of the stress tensor which map to the Virasoro module of the identity of the CFT.

As a consequence, if the degrees of freedom were only those of the identity module, pure gravity would not admit a quantum completion. The reason is very simple: The partition function of the CFT on the toroidal boundary is modular invariant, while the character of the identity module, as well as any other single Virasoro character, is not. Thus modular invariance of the boundary theory implies additional degrees of freedom in the bulk.

What is the meaning of modular invariance on the gravity side? In a quantum approach to gravity one expects to sum over different topologies of space-time with fixed asymptotic boundary conditions. It is widely believed that modular invariance arises from the sum over saddle points of the gravitational path integral [2-4]. One such geometry is thermal AdS with periodic Euclidean time. Others correspond to the Euclidean black holes discovered by Bañados, Teitelboim,

Published by the American Physical Society under the terms of the Creative Commons Attribution 4.0 International license. Further distribution of this work must maintain attribution to the author(s) and the published article's title, journal citation, and DOI. and Zanelli in any three-dimensional gravity with negative cosmological constant [5]. Thus Bañados-Teitelboim-Zanelli (BTZ) black holes are necessary degrees of freedom for a quantum description of pure gravity in asymptotic $\mathrm{AdS}_{3}$ space-times. Are these enough? BTZ black holes can exist only above some mass threshold that in the large- $c$ limit is holographically dual to a CFT primary of scaling dimension $\Delta_{\mathrm{BTZ}}=\frac{c-1}{12}$, where $c$ is the central charge.

Primary operators with $\Delta<\Delta_{\mathrm{BTZ}}$ cannot be interpreted as black holes; they correspond to a different kind of matter (this point will be discussed in more detail later). Therefore, proving that a primary with $\Delta<\Delta_{\mathrm{BTZ}}$ is necessary for a consistent CFT at the boundary would be sufficient to argue that pure quantum gravity does not exist.

Modular invariance of the partition function constrains the possible spectra of 2D CFTs [6]. In particular, as first pointed out by Hellerman [7], for general unitary 2D CFTs with $c>1$ it is possible to find an upper bound for the allowed scaling dimension, $\Delta_{0}$, of the first nontrivial primary. Hellerman rigorously established the inequality $\Delta_{0}<\frac{c}{6}+0.4737$. This bound has since been improved numerically as well as analytically in various ways [8-15], in particular using the linear programming method introduced in [16]. So far, in the large- $c$ limit the best analytic bound is $\Delta_{0} \leqslant c / 8.503$ [13], while the numerical upper bound, obtained by extrapolating large- $c$ data, is $\Delta_{0} \leqslant c / 9.08$ [12]. Other bounds can be found by assuming complete factorization of the partition function into a holomorphic and an antiholomorphic part [17]. In this case, the CFT includes an infinite set of conserved higher spin currents, while it is widely believed that pure gravity is dual to a CFT in which the only conserved currents are those generated by the stress tensor.

The modular group of the torus $\operatorname{PSL}(2, \mathbb{Z})$ is the group of linear fractional transformations acting on the modular parameter $\tau \in H_{+}\left(H_{+}\right.$is the upper half plane) and generated by the inversion $S$ and the translation $T$, satisfying 
$S^{2}=(S T)^{3}=1$. One of the reasons why the modular invariance of the partition function $Z$ is so constraining is that $\operatorname{PSL}(2, \mathbb{Z})$ does not act freely on $H_{+}$: There are points of $H_{+}$ which are left invariant under the action of some nontrivial subgroup of $\operatorname{PSL}(2, \mathbb{Z})$. The partition function is a smooth function of $\tau$ only if it fulfills certain consistency conditions at these special points. $\operatorname{PSL}(2, \mathbb{Z})$ admits three points of this kind. So far, only two of them have been extensively explored in modular bootstrap studies. The third one, left invariant by $S T$, has been considered so far only by Qualls [10], who wrote a set of consistency equations, under the assumption that there are partition functions in which only even-spin primaries contribute.

In this paper we considerably enlarge the set of equations considered by Qualls. These give rise to a wealth of information about infinitely many primary operators for any 2D CFT with central charge $c>1$. Equations (14) show some examples of them, written as simple sum rules involving both the spins and the scaling dimensions of primary operators. In particular, the second of Eqs. (14) clearly excludes the existence of unitary CFTs with only even-spin primaries for any $c>1$, in contrast to the assumption of [10].

The large- $c$ limit of these sum rules is especially interesting because unitary CFTs with large $c$ are holographically dual to quantum gravity in asymptotically $\mathrm{AdS}$ space-times. In this limit we derive from them a tighter upper bound on the allowed scaling dimensions of the first nontrivial primary, namely,

$$
\Delta_{0}<\frac{c-1}{12}+\frac{1}{2 \sqrt{3} \pi},
$$

which is far stronger than those found to date and is valid under the same assumptions of [7], with the further specification that the scaling dimension of the first odd-spin primary must lie below $\Delta_{0}$.

It is worth stressing that this bound is remarkably close to the threshold $\Delta_{\mathrm{BTZ}}=\frac{c-1}{12}$, which on the gravitational side constrains the minimal mass of black holes. In addition, our derivation shows that this upper bound is not an extremum, suggesting it should be possible to improve it further. Even a modest improvement could push $\Delta_{0}$ down to the BTZ threshold, implying that pure Einstein gravity in $\mathrm{AdS}_{3}$ does not exist as a quantum theory.

\section{CONSTRAINTS FROM MODULAR INVARIANCE}

Modular invariance of the partition function is guaranteed to be a universal property of any physically meaningful theory formulated on a torus, since modular transformations correspond to changes of the basis on $\mathbb{Z}+\tau \mathbb{Z}$, its period lattice, while the physics cannot depend on the choice of this basis. It reads

$$
Z(\tau, \bar{\tau})=Z\left(\frac{a \tau+b}{c \tau+d}, \frac{a \bar{\tau}+b}{c \bar{\tau}+d}\right), \quad\left(\begin{array}{ll}
a & b \\
c & d
\end{array}\right) \in \operatorname{PSL}(2, \mathbb{Z}) .
$$

Here $\tau$ and $\bar{\tau}$ may be considered as two independent complex variables with $\tau \in H_{+}$and $\bar{\tau} \in H_{-}$, where $H_{+}$and $H_{-}$are the upper and the lower half planes. In addition, $Z(\tau, \bar{\tau})$ becomes the partition function on a torus of modular parameter $\tau$ when $\bar{\tau}$ is chosen to be the complex conjugate of $\tau$, but Eq. (2) is more general. If the theory on the torus is conformally invariant, we can expand $Z$ as a sum over all contributing states

$$
Z(\tau, \bar{\tau})=\sum_{h, \bar{h}} q^{h-c / 24} \bar{q}^{\bar{h}-c / 24}, \quad q=e^{2 i \pi \tau}, \quad \bar{q}=e^{-2 i \pi \bar{\tau}}
$$

where $\Delta=h+\bar{h}$ are the scaling dimensions of the states and $j=h-\bar{h}$ their spins. For the sake of simplicity, we have taken the same central charge $c$ for the left and the right Virasoro algebras. There is a unique vacuum with $h=\bar{h}=0$. If one further assumes the theory unitary with a discrete spectrum, then Eq. (3) implies that $Z$ is a holomorphic function on $H_{+} \times H_{-}$.

The partition function of any physical theory formulated on a torus is assumed to be a smooth function on the fundamental domain $H_{+} / \operatorname{PSL}(2, \mathbb{Z})$ [or $\left.H_{-} / \operatorname{PSL}(2, \mathbb{Z})\right]$. We can then apply a property which is at the core of modular bootstrap [6,7]: A smooth function on the fundamental domain lifts to a smooth function on its covering space $H_{+}$(or $H_{-}$) if and only if it satisfies certain consistency conditions on its derivatives at the elliptic points and at the cusp, i.e., the special points of the fundamental region which are invariant under the action of some nontrivial subgroup of $\operatorname{PSL}(2, \mathbb{Z})$.

As already mentioned in the Introduction, the torus modular group admits three points of this kind. The cusp at $\tau=i \infty$ is stabilized by the subgroup generated by $T: \tau \rightarrow \tau+1$. The $\mathbb{Z}_{2}$ elliptic point at $\tau=i$ is stabilized by $S: \tau \rightarrow-1 / \tau$. The elliptic point at $\tau=e^{2 i \pi / 3}$ is stabilized by $S T: \tau \rightarrow$ $\frac{-1}{\tau+1}$, the generator of a $\mathbb{Z}_{3}$ subgroup of $\operatorname{PSL}(2, \mathbb{Z})$.

Invariance of the partition function under $T$ implies integer spins, i.e., $j=h-\bar{h} \in \mathbb{Z}$, and analyticity at the cusp $\tau=i \infty$ yields, for $c>1$, the upper bound $\Delta-|j| \leqslant \frac{c-1}{12}$ on the twist gap [11]. Consistency conditions at $\tau=i$ demand [7]

$$
\left.\left(\tau \partial_{\tau}\right)^{m}\left(\bar{\tau} \partial_{\bar{\tau}}\right)^{n} Z(\tau, \bar{\tau})\right|_{\tau=-\bar{\tau}=i}=0 \quad \text { for } m+n \text { odd } .
$$

This infinite set of homogeneous linear equations, dubbed a modular bootstrap, yields the Hellerman upper bound on the scaling dimensions of the lightest primary operator and its refinements described in the Introduction.

What are the further constraints dictated by the consistency conditions at the point stabilized by $S T$ ? It suffices to take arbitrary derivatives of the identity

$$
Z(\tau, \bar{\tau})=Z\left(\frac{-1}{\tau+1}, \frac{-1}{\bar{\tau}+1}\right)
$$

and evaluate them at $\tau=e^{2 i \pi / 3} \equiv \rho$. We obtain

$$
\left.\partial_{\tau}^{n} Z\right|_{\tau=\rho}-\left.\sum_{m=1}^{n} \rho^{m+n} \frac{n !}{m !}\left(\begin{array}{c}
n-1 \\
m-1
\end{array}\right) \partial_{\tau}^{m} Z\right|_{\tau=\rho}=0
$$

and identical equations for $\bar{\tau}$. Since $\rho^{3 k}=1$, iterating the above equations shows that a derivative of arbitrary order in $\tau$ (or in $\bar{\tau}$ ) at $\tau=\rho$ (or $\bar{\tau}=\bar{\rho}$ ) can be expressed as a linear combination of derivatives of lower order in multiples of 3 . These are the equations to be added to (4) to complete the modular bootstrap program. 
In order to keep $Z$ real it is convenient to parametrize $\tau$ and $\bar{\tau}$ as

$$
\tau=-\frac{1}{2}+i \frac{\beta}{2 \pi}, \quad \bar{\tau}=-\frac{1}{2}-i \frac{\bar{\beta}}{2 \pi},
$$

where $\beta$ and $\bar{\beta}$ are two independent real variables, so we can set $q=-e^{-\beta}$ and $\bar{q}=-e^{-\bar{\beta}}$ in (3), thus obtaining a real partition function. Terms with even $j=h-\bar{h}$ are positive while those with odd $j$ are negative. The $\mathbb{Z}_{3}$ elliptic point corresponds to $\beta=\bar{\beta}=\sqrt{3} \pi \equiv \beta_{c}$.

The first few equations are, more explicitly,

$$
\begin{array}{r}
\left.\partial_{\beta} Z\right|_{\beta=\bar{\beta}=\beta_{c}}=0,\left.\quad\left(\partial_{\beta}^{4}+\frac{2 \sqrt{3}}{\pi} \partial_{\beta}^{3}\right) Z\right|_{\beta=\bar{\beta}=\beta_{c}}=0, \\
\left.\partial_{\beta}^{2} Z\right|_{\beta=\bar{\beta}=\beta_{c}}=0,\left.\quad\left(\partial_{\beta}^{5}-\frac{10}{\pi^{2}} \partial_{\beta}^{3}\right) Z\right|_{\beta=\bar{\beta}=\beta_{c}}=0, \\
\left.\left(\partial_{\beta}^{7}+\frac{525}{\pi^{4}} \partial_{\beta}^{3}+\frac{7 \sqrt{3}}{\pi} \partial_{\beta}^{6}\right) Z\right|_{\beta=\bar{\beta}=\beta_{c}}=0, \\
\left.\left(\partial_{\beta}^{8}-\frac{1470 \sqrt{3}}{\pi^{5}} \partial_{\beta}^{3}-\frac{98}{\pi^{2}} \partial_{\beta}^{6}\right) Z\right|_{\beta=\bar{\beta}=\beta_{c}}=0,
\end{array}
$$

with identical equations for $\bar{\beta}$. One can check these identities by applying them, for instance, to the modular invariant

$$
\sqrt{\tau-\bar{\tau}} \eta(\tau) \eta(-\bar{\tau})=Z_{b}^{-1},
$$

where $Z_{b}$ is the partition function of a free boson and $\eta$ is the Dedekind eta function.

In order to obtain useful information on the Virasoro primary spectrum, we have to separate primaries from descendants in the sum of states (3). The $Z(\tau, \bar{\tau})$ can be expanded in Virasoro characters. If $c>1$ and the theory is unitary, the modules of the Virasoro algebra are the identity degenerate module $\chi_{0}(q)$ and a continuous family of nondegenerate modules $\chi_{A}(q)$ labeled by a positive conformal weight $h_{A}$,

$$
\chi_{0}(q)=\frac{q^{-(c-1) / 24}}{\eta(\tau)}(1-q), \quad \chi_{A}(q)=\frac{q^{h_{A}-(c-1) / 24}}{\eta(\tau)} .
$$

Assuming discreteness of the spectrum and no conserved currents beyond those of the Virasoro algebra yields the expansion $Z=\left[0_{0}\right]+\sum_{A} N_{A}\left[\Delta_{j_{A}}\right]$, or more explicitly

$$
Z(\tau, \bar{\tau})=\chi_{0}(q) \chi_{0}(\bar{q})+\sum_{A} N_{A} \chi_{A}(q) \chi_{A}(\bar{q}),
$$

where the multiplicity $N_{A}$ is a non-negative integer.

In order to obtain the promised upper bound it suffices to apply to such an expansion the first few identities (8). We get rid of the $\eta$ function and its derivatives by considering the modular-invariant combination $Z(\tau, \bar{\tau}) / Z_{b}=Z_{\mathrm{vac}}+$ $\sum_{A} N_{A} Z_{A}$, with

$$
\begin{aligned}
Z_{\mathrm{vac}} & =\sqrt{\beta+\bar{\beta}} e^{\beta[(c-1) / 24]} e^{\bar{\beta}[(c-1) / 24]}\left(1+e^{\beta}\right)\left(1+e^{\bar{\beta}}\right), \\
Z_{A} & =\sqrt{\beta+\bar{\beta}} e^{\beta[(c-1) / 24]} e^{\bar{\beta}[(c-1) / 24]} e^{-\beta h_{A}} e^{-\bar{\beta} \bar{h}_{A}} .
\end{aligned}
$$

When applying (8) it turns out that the scaling dimensions $\Delta_{A}=h_{A}+\bar{h}_{A}$ always appear in the combination $\Delta_{A}-\Delta_{+}$ with

$$
\Delta_{+}=\frac{c-1}{12}+\frac{1}{2 \sqrt{3} \pi} .
$$

It is convenient to organize Eqs. (8) in terms of polynomials of the two differential operators $\partial_{\beta}+\partial_{\bar{\beta}}$ and $\partial_{\beta}-\partial_{\bar{\beta}}$. Odd powers of the latter give trivial identities as a consequence of the symmetry of the partition function under the transformation $j_{A} \rightarrow-j_{A}$. The other identities can be written as surprisingly simple sum rules; the first few, corresponding, respectively, to the operators $\partial_{\beta}+\partial_{\bar{\beta}},\left(\partial_{\beta}-\partial_{\bar{\beta}}\right)^{2}$, and $\left(\partial_{\beta}+\right.$ $\left.\partial_{\bar{\beta}}\right)^{2}$, are

$$
\begin{aligned}
& \sum_{A}(-1)^{j_{A}} N_{A} e^{-\beta_{c} \Delta_{A}}\left(\Delta_{A}-\Delta_{+}\right)=v^{2} \Delta_{+}-u v, \\
& \sum_{A}(-1)^{j_{A}} N_{A} e^{-\beta_{c} \Delta_{A}} j_{A}^{2}=-u, \\
& \sum_{A}(-1)^{j_{A}} N_{A} e^{-\beta_{c} \Delta_{A}}\left(\left(\Delta_{A}-\Delta_{+}\right)^{2}-\frac{1}{6 \pi^{2}}\right) \\
& \quad=-v^{2} \Delta_{+}^{2}+2 u v \Delta_{+}-u(1+u)+\frac{v^{2}}{6 \pi^{2}},
\end{aligned}
$$

with $u=2 e^{-\beta_{c}}$ and $v=1+e^{-\beta_{c}}$. The left-hand sides of these sum rules measure the difference in contributions of even and odd spins. Those considered by Qualls in [10] correspond to set $\beta=\bar{\beta}$ before taking the derivatives. As already mentioned in the Introduction, the possibility suggested in [10] that there exist theories where only primaries of even spin contribute is excluded by the second of Eqs. (14) for any unitary CFT with $c>1$. We expect that at large $j$ the two kinds of contributions cancel, as the density of the states is the same in this limit [18-20]; however, we do not use such a cancellation in deriving our upper bound.

We can check the above equations in some specific model. An instructive example is the partition function $Z_{f}$ of eight free fermions with diagonal Gliozzi-Scherk-Olive projection, which saturates the unitarity bound at $c=4[11,13]$. The full partition function reads

$$
Z_{f}(\tau)=\sum_{i=2}^{4} \frac{\theta_{i}(\tau)^{4} \theta_{i}(-\bar{\tau})^{4}}{2 \eta(\tau)^{4} \eta(-\bar{\tau})^{4}},
$$

where $\theta_{i}$ denotes the Jacobi theta function. Further, $Z_{f}$ vanishes for $\tau \rightarrow e^{2 i \pi / 3}$, as it becomes proportional to the Eisenstein series $E_{4}(\tau)$, which is known to have a simple zero at this point, so in this case the cancellation is complete. In our notation the vanishing of $Z_{f}$ reads $^{1}$

$$
\sum_{A}(-1)^{j_{A}} N_{A} e^{-\beta_{c} \Delta_{A}}=-v^{2} .
$$

We can easily expand $Z_{f}$ in Virasoro characters. The first few terms are

$$
\begin{aligned}
Z_{f}= & {\left[0_{0}\right]+28\left[1_{1}\right]+192\left[1_{0}\right]+105\left[2_{2}\right]+1344\left[2_{1}\right] } \\
& +784\left[2_{0}\right]+\cdots .
\end{aligned}
$$

\footnotetext{
${ }^{1}$ The same identity holds for the partition function of eight compactified bosons on the $\Gamma_{8}$ Narain lattice mentioned in [11].
} 
This expansion includes conserved currents, i.e., primaries of the form $\left[j_{j}\right]$ that we had excluded in deriving (14). The contribution of a conserved current to the partition function is $\chi_{j}(q) \chi_{0}(\bar{q})+\chi_{0}(q) \chi_{j}(\bar{q})$. One can repeat the calculation that led to (14) and check that the consequent modification is numerically negligible. As a matter of fact, the two-level decomposition (17) is enough to give a good numerical check of the first of Eqs. (14), which involves only first derivatives, while the others require much more terms.

Equations (14) and their analogs with higher derivatives become particularly interesting in the large- $c$ limit, where they simply read, for integer $n$,

$$
\sum_{A}(-1)^{j_{A}} N_{A} e^{-\beta_{c} \Delta_{A}}\left(\frac{\Delta_{A}-\Delta_{+}}{\Delta_{+}}\right)^{n}=v^{2}(-1)^{n+1}+O\left(\frac{1}{c}\right) .
$$

We arrange the terms of these series following the increasing order of the scaling dimensions of the primaries, i.e., $0<$ $\Delta_{1} \leqslant \Delta_{2} \leqslant \cdots$, and denote by $S_{n}^{(k)}$ the partial sum of the first $k$ terms of the $n$th series. We assume the convergence of these series. This means that for every real number $\epsilon>$ 0 there exists an integer $m_{n}$ such that for all $k \geqslant m_{n}$ we have $\left|S_{n}^{(k)}-S_{n}\right|<\epsilon$, where $S_{n} \equiv v^{2}(-1)^{n+1}$ is the sum of the series. Choosing $\epsilon$ small enough implies that all the finite sums $S_{n}^{(k)}$ with $k \geqslant m_{n}$ have the same sign as $S_{n}$. Thus, from the convergence of the first $n_{0}$ series we obtain the exact $n_{0}$ inequalities $S_{n=2 \ell-1}^{(m)}>0$ and $S_{n=2 \ell}^{(m)}<0$, with $m=\max \left(m_{n}\right)$ $\left(1 \leqslant n \leqslant n_{0}\right)$. We recast the above inequalities in the form

$$
\begin{aligned}
A_{n} & >B_{n} \quad \text { if } n \text { odd } \\
& <B_{n} \quad \text { if } n \text { even, }
\end{aligned}
$$

where to simplify the notation we defined $A_{n}=\sum_{i=1}^{p} w_{i} a_{i}^{n}$ and $B_{n}=\sum_{j=1}^{q} z_{j} b_{j}^{n}$, with $p+q=m$; the variables $a_{1}<$ $a_{2}<\cdots<a_{p}$ and $b_{1}<b_{2}<\cdots<b_{q}$ denote the even-spin and the odd-spin terms, respectively, and $w_{i}>0$ and $z_{j}>0$ their multiplicity.

So far we only assumed the convergence of the series. If we now assume a stronger hypothesis, namely, that the first $n_{0}$ series, for a suitable $n_{0}$, fulfills the condition

$$
m \equiv p+q<n_{0},
$$

we can easily demonstrate that the scaling dimension of the lowest odd-spin primary lies below $\Delta_{+}$, i.e., $b_{1}<0$.

This theorem can be proved by reductio ad absurdum, i.e., assuming $b_{1}>0$, we show that the inequalities (19) have no solution. First, note that some of the $a_{i}$ 's could be negative, so their exclusion reinforces the inequalities, and thus we also assume $a_{i}>0$. It is easy to see that the $A$ 's (and the $B$ 's) fulfill a linear relation of the form $\sum_{j=0}^{p} \lambda_{j} A_{p+k-j}=0$ $(k=1,2, \ldots)$, which can be rewritten as

$$
\sum_{i=1}^{p} w_{i}\left(\sum_{j=0}^{p} \lambda_{j} a_{i}^{p+k-j}\right)=0 .
$$

To prove this identity, it suffices to point out that the $\lambda_{j}$ 's are the coefficients of the polynomial $x^{k} \prod_{j=1}^{p}\left(x-a_{j}\right) \equiv$ $x^{k}\left(\sum_{j=0}^{p} \lambda_{j} x^{p-j}\right)$, with $\lambda_{0}=1$.
Since all the $a_{i}$ 's are positive, the $\lambda_{j}$ have alternating signs, i.e., $\lambda_{1}<0, \lambda_{2}>0$, and so on; therefore, applying the inequalities (19) to the identity (21) gives

$$
\begin{aligned}
B_{p+k}+\lambda_{1} B_{p+k-1}+\cdots+\lambda_{p} B_{k}<0 & \text { if } p+k \text { odd } \\
& >0 \quad \text { if } p+k \text { even. }
\end{aligned}
$$

In accordance with the assumption (20), $k$ can take the values $k=1,2, \ldots, q+1$.

We rewrite the left-hand side of these $q+1$ inequalities as $\sum_{j=1}^{q} y_{j} b_{j}^{k}$, with $y_{j}=z_{j} \prod_{i=1}^{p}\left(b_{j}-a_{i}\right)$. Clearly the alternating signs of (22) are possible only if there are both positive and negative $y_{j}$ 's. Following the signs of $y_{j}$, we split the set of the $q$ variables $b_{j}$ into two subsets of $p^{\prime}$ and $q^{\prime}$ elements with $q=p^{\prime}+q^{\prime}$. In this way, relabeling the indices, we can recast the $q+1$ inequalities (22) into the same form as (19), but with a reduced number of variables.

The iteration of this process terminates when we are left with a single variable of type $b$ and $q^{\prime}+1=2$ contradictory inequalities, namely, $b>0$ and $b^{2}<0$, showing that the set (19) combined with (20) is incompatible with the assumption $b_{1}>0$. Q.E.D.

On the contrary, allowing $b_{1}$ to be negative, it is easy to find numerical solutions of (19). Some of them also have negative even-spin terms, suggesting a possible improvement of the upper bound.

\section{DISCUSSION AND OUTLOOK}

In this paper we pointed out that the partition function of a general CFT on a torus should obey a large class of equations. The equations allow us to derive a strong upper bound for the maximal gap of the first nontrivial primary in the large- $c$ limit. The latter is very close to the mass threshold of the corresponding BTZ black holes in $\mathrm{AdS}_{3} / \mathrm{CFT}_{2}$ correspondence.

For the sake of completeness, let us recall how such a threshold emerges in the holographic approach. A BTZ black hole of mass $M$ and spin $j$ in the bulk corresponds to a $(h, \bar{h})$ primary on the boundary with

$$
h-\frac{c}{24}=\frac{1}{2}(M \ell+j), \quad \bar{h}-\frac{c}{24}=\frac{1}{2}(M \ell-j),
$$

where $\ell$ is the radius of $\mathrm{AdS}_{3}$ space-time, related to the central charge in [21] $c=\frac{3 \ell}{2 G}$, with $G$ the Newton constant. Black holes have smooth horizons only if they fulfill the cosmic censorship condition $M \ell \geqslant|j|$, prohibiting naked singularities in space-time. Therefore, according to (23), a CFT primary corresponds to a black hole in the bulk only if $h, \bar{h} \geqslant \frac{c}{24}$. One-loop corrections replace $c$ with $c-1$ [15]. Primaries with $\Delta<\frac{c-1}{12}$ correspond to objects that do not have a smooth event horizon, the defining feature of black holes, and thus should correspond to a different kind of matter in the bulk.

The threshold at $\Delta=\frac{c-1}{12}$ has a special meaning even on the CFT side [15,22]. In particular, in a unitary CFT with a boundary, a lower bound is derived for the boundary entropy when assuming $\Delta_{0}>\frac{c-1}{12}$ [22]; however, lower values are possible. Actually, some indication that a lower upper bound is necessary for saving unitarity has been found recently [14], since in the double limit $j \rightarrow \infty$ and $\bar{h}-\frac{c-1}{24} \rightarrow 0$ the density 
of states of the vacuum character contribution $\chi_{0}(q) \chi_{0}(\bar{q})$ becomes negative. A possibility to avoid this violation of unitarity is assuming a twist gap no larger than $\frac{c-1}{16}$.

From this perspective, it would be interesting to try to improve our upper bound (1). Note that to derive it only some simple properties of the set of equations (6) were used. A further study of them could generate important information on the spectrum of primary states in a general CFT.

\section{ACKNOWLEDGMENT}

The author acknowledges a fruitful correspondence with Slava Rychkov.
[1] J. M. Maldacena, The Large-N limit of superconformal field theories and supergravity, Int. J. Theor. Phys. 38, 1113 (1999) [Adv. Theor. Math. Phys. 2, 231 (1998)].

[2] R. Dijkgraaf, J. M. Maldacena, G. W. Moore, and E. P. Verlinde, A black hole Farey tail, arXiv:hep-th/0005003.

[3] J. Manschot and G. W. Moore, A modern Farey tail, Commun. Number Theor. Phys. 4, 103 (2010).

[4] C. A. Keller and A. Maloney, Poincaré series, 3D gravity and CFT spectroscopy, J. High Energy Phys. 02 (2015) 080.

[5] M. Bañados, C. Teitelboim, and J. Zanelli, Black Hole in ThreeDimensional Spacetime, Phys. Rev. Lett. 69, 1849 (1992).

[6] J. L. Cardy, Operator content of two-dimensional conformally invariant theories, Nucl. Phys. B 270, 186 (1986).

[7] S. Hellerman, A universal inequality for CFT and quantum gravity, J. High Energy Phys. 08 (2011) 130.

[8] D. Friedan and C. A. Keller, Constraints on 2d CFT partition functions, J. High Energy Phys. 10 (2013) 180.

[9] T. Hartman, C. A. Keller, and B. Stoica, Universal spectrum of $2 \mathrm{~d}$ conformal field theory in the large $c$ limit, J. High Energy Phys. 09 (2014) 118.

[10] J. D. Qualls, Universal bounds in even-spin CFTs, J. High Energy Phys. 12 (2015) 001.

[11] S. Collier, Y. H. Lin, and X. Yin, Modular bootstrap revisited, J. High Energy Phys. 09 (2018) 061.

[12] N. Afkhami-Jeddi, T. Hartman, and A. Tajdini, Fast conformal bootstrap and constraints on 3d gravity, J. High Energy Phys. 05 (2019) 087.

[13] T. Hartman, D. Mazáč, and L. Rastelli, Sphere packing and quantum gravity, J. High Energy Phys. 12 (2019) 048.
[14] N. Benjamin, H. Ooguri, S. H. Shao, and Y. Wang, Lightcone modular bootstrap and pure gravity, Phys. Rev. D 100, 066029 (2019).

[15] H. Maxfield, Quantum corrections to the BTZ black hole extremality bound from the conformal bootstrap, J. High Energy Phys. 12 (2019) 003.

[16] R. Rattazzi, V. S. Rychkov, E. Tonni, and A. Vichi, Bounding scalar operator dimensions in 4D CFT, J. High Energy Phys. 12 (2008) 031.

[17] E. Witten, Three-dimensional gravity revisited, arXiv:0706.3359.

[18] Y. Kusuki, Light cone bootstrap in general 2D CFTs and entanglement from light cone singularity, J. High Energy Phys. 01 (2019) 025.

[19] S. Collier, Y. Gobeil, H. Maxfield, and E. Perlmutter, Quantum Regge trajectories and the Virasoro analytic bootstrap, J. High Energy Phys. 05 (2019) 212.

[20] Y. Kusuki and M. Miyaji, Entanglement entropy, OTOC and bootstrap in 2D CFTs from Regge and light cone limits of multi-point conformal block, J. High Energy Phys. 08 (2019) 063.

[21] J. D. Brown and M. Henneaux, Central charges in the canonical realization of asymptotic symmetries: An example from three-dimensional gravity, Commun. Math. Phys. 104, 207 (1986).

[22] D. Friedan, A. Konechny, and C. Schmidt-Colinet, Lower Bound on the Entropy of Boundaries and Junctions in $(1+1)$ Dimensional Quantum Critical Systems, Phys. Rev. Lett. 109, 140401 (2012). 